The Astrophysical Journal, 190:L63-L66, 1974 June 1

(c) 1974. The American Astronomical Society. All rights reserved. Printed in U.S.A.

\title{
NONPERIODIC OPTICAL FLICKERING IN HZ HERCULIS
}

\author{
T. J. Moffett, R. E. Nather, and P. A. Vanden Bout \\ The University of Texas at Austin and McDonald Observatory \\ Received 1974 January 25; revised 1974 March 27
}

\begin{abstract}
High-speed simultaneous dual-channel photometry of $\mathrm{HZ}$ Her and a nearby comparison star reveal nonperiodic optical flickering in the HZ Her system on a time scale of 15-300 s. The amplitude of the flickering appears to be correlated with orbital phase. Optical emission from a hot spot in a disk of material around the X-ray source cannot account for the flickering.

Subject headings: photometry $-\mathrm{X}$-ray sources
\end{abstract}

\section{INTRODUCTION}

A number of observational and theoretical considerations suggest the presence of "third light" in the $\mathrm{HZ}$ Her/Her X-1 system. Boynton et al. (1973) require a hot component near maximum light to account for their $U B V r$ observations. Bopp et al. $(1972,1973)$ find rapid variations in the $\lambda \lambda 4650,4686$ emission lines. According to Joss, Avni, and Bahcall (1973) and Pringle (1973), the fact that the optical light curve is sharper and narrower than the X-ray intensity curve near minimum is due to light that originates well above the photosphere of HZ Her. Strittmatter et al. (1973) have a model for this system involving a significant contribution to the total light by emission from an accretion disk surrounding Her X-1. These properties are reminiscent of the cataclysmic variable stars. In an attempt to see whether the models developed for these stars by Smak (1971) and Warner and Nather (1971) could be applied to the HZ Her system, we have tried to detect nonperiodic optical flickering, common in cataclysmic variables, in the light curve of $\mathrm{HZ}$ Her.

This effort has been successful in that HZ Her does show nonperiodic variations on a time scale of $15-300 \mathrm{~s}$ which are appreciably larger than the statistical and scintillation noise. However, the variation in amplitude of this flickering as a function of orbital phase rules out the hot-spot-accretion-disk model that accounts for the flickering in the cataclysmic variables. The observations suggest that the "third light" originates near $\mathrm{HZ}$ Her on the hot side toward Her X-1 and might be the result of fluctuations in X-ray heating.

\section{OBSERVATIONS AND ANALYSIS}

Our observations were obtained with the 82-inch $(208-\mathrm{cm})$ Struve reflector of the McDonald Observatory, using the dual-channel pulse-counting photometer (Nather 1972). No filter was inserted in the optical train, so the short-wavelength cutoff was determined primarily by atmospheric extinction, while the response at longer wavelengths was limited by the bi-alkali photocathodes of the photomultiplier tubes used. The instru- ment was operated as a simultaneous two-star photometer, with the variable normally measured by the Amperex 56 DVP phototube in channel 1, while a nearby comparison star (located $\sim 6^{\prime} \mathrm{W}$ and $1^{\prime} \mathrm{N} ; V=$ 12.58, $B-V=+0.58, U-B=+0.03$ ) was monitored by the RCA 4516 phototube in channel 2. The comparison star was also used for guiding, so its photometric record was interrupted for one or two readings at intervals of roughly 5 minutes to permit guiding corrections. With this exception photometric measurement was continuous, the readings being divided into contiguous 4-s integrations.

The two separate apertures used were aligned by allowing the two stars to drift through them slowly, while recording the intensities of the two channels. The resulting "light curve" was an accurate measure of the relative sizes and positions of the two apertures. The iris diaphragm in the second channel was carefully adjusted so that it was slightly smaller than, and everywhere inside, the diaphragm in channel 1 to ensure that any guiding errors or seeing difficulties would be recorded in the monitor channel for later editing. This procedure has been adopted as standard for all twochannel measurements with this instrument.

The technique of simultaneous two-star measurement permits a direct comparison of the light curves of the two objects, in addition to certifying the reality of small changes in the variable star. Flickering was observed earlier in $\mathrm{HZ} \mathrm{Her} \mathrm{on} \mathrm{the} \mathrm{same} \mathrm{time} \mathrm{scale} \mathrm{reported}$ here, but without the simultaneous measurement of a comparison star; since such changes might be due to changing sky conditions or other factors, their reality was not unassailable. Earlier attempts (Groth and Nelson 1972) to detect nonperiodic variations in $\mathrm{HZ} \mathrm{Her}$ met with similar difficulties.

All of the observations were corrected for sky background, and the effects of atmospheric extinction were removed by the application of mean extinction coefficients appropriate for McDonald Observatory. The corrected data showed slow changes in level common to both variable and comparison stars, apparently the effect of variable transparency. To remove these effects, 
' we first smoothed the comparison star data by the ap: plication of a four-point running (16-s) square filter, ini sufficiently long to minimize the high-frequency stoIHI chastic and scintillation noise present but short compared with the transparency changes, and then divided both the variable and comparison star data by this smoothed curve. The resulting light curves for both stars are shown in figure 1 , identified in orbital phase from the elements $J D_{\odot}=2,441,644.10587+1 \mathrm{~d} 700165 \mathrm{E}$

In all cases the variations in the light curve of $\mathrm{HZ} \mathrm{Her}$ clearly exceed those of the comparison star, which had nearly the same detected photon arrival rate as the variable, showing that flickering activity was present at the three orbital phases examined.
Cataclysmic variables exhibit a wide variety of flickering activity arising from the mass transfer process, and in many cases the amplitude of this activity is a strong function of orbital phase. To search these data for similar behavior we must first remove the effects of other sources of variation; in this case, since the two stars had comparable counting rates, the contribution from photon arrival noise was essentially the same for both, and contributions from dark noise and from the sky were negligible. Scintillation noise contributed materially to the apparent variations in all of the runs, however, and was of differing intensity on different nights. If we assume that the comparison star has no intrinsic variations of its own, we can use its light curve,
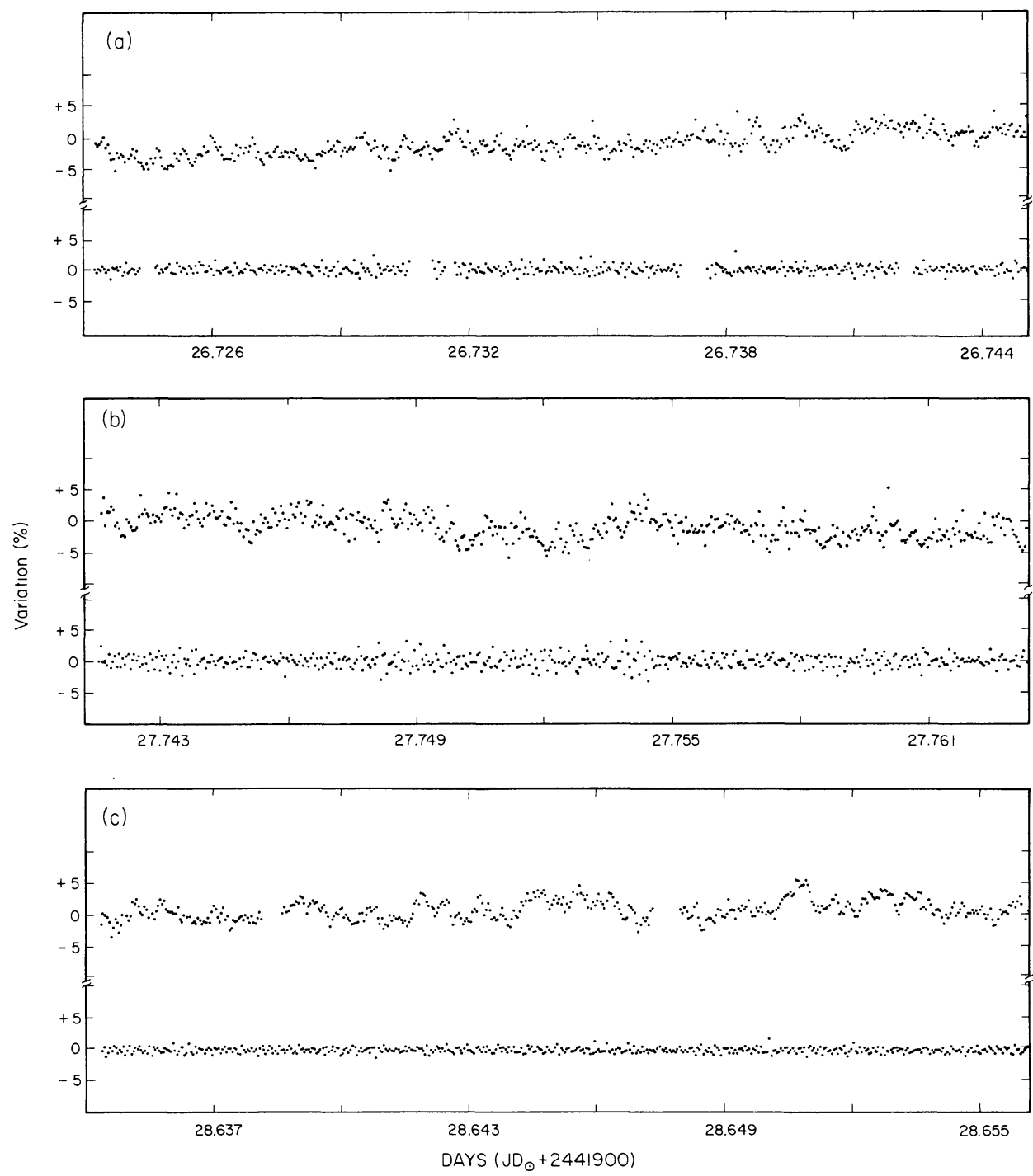

Fig. 1.-Light curves of HZ Her (upper) and a nearby comparison star (lower) for orbital phases: $(a) \Phi=0.229,(b) \Phi=0.835$, and $(c) \Phi=0.372$. The curves have been normalized by the techniques described in the text. $\mathrm{JD}_{\odot} 2,441,928.637=1973$ September $0303^{\mathrm{h}} 17^{\mathrm{m}} 12^{\mathrm{e}}$ UT. 
properly scaled, as a measure of the contribution from scintillation noise to the total observed variations.

On the assumption that the percentage modulation from scintillation effects is the same for all stars-i.e., that the noise from this source is proportional to the intensity of the measured starlight - then variance of the variable star data $\left(\sigma_{F}^{2}\right)$ due only to intrinsic variations is given by

$$
\sigma_{F}^{2}=\sigma_{\mathrm{Hz}}^{2}-N_{\mathrm{HZ}}-\left(N_{\mathrm{Hz}} / N_{C}\right)^{2}\left(\sigma_{C}^{2}-N_{C}\right),
$$

where $\sigma_{\mathrm{Hz}}{ }^{2}$ is the total variance observed; $N_{\mathrm{Hz}}$ and $N_{C}$ are the counting rates of $\mathrm{HZ}$ Her and the comparison star, respectively; and $\sigma_{C}{ }^{2}$ is the variance of the comparison-star data.

The net intensity of the flickering in $\mathrm{HZ} \mathrm{Her,} \mathrm{ex-}$ pressed as a percentage of the total light $\left(F=\sigma_{F} / N_{\mathrm{HZ}}\right)$ is shown, together with computed orbital phase, in table 1. While the data are too sparse to draw strong conclusions concerning the variation of the flickering with orbital phase, we note that it seems appreciably higher at phase 0.37 than at the other phases, and may have a maximum near phase 0.5 .

We also obtained data at integration times of $0.25 \mathrm{~s}$ (also listed in table 1) to permit a search for optical pulsations related to the 1.23-s X-ray period; powerspectrum analysis of these data show no significant periodicities present at or near the expected frequency. This is consistent with the suggestion that the optical pulsing seems to be confined to orbital phases near $\Phi=0.25$ and $\Phi=0.75$ (Davidsen et al. 1972).

Just before or after each of the runs in table 1 we also observed HZ Her through an interference filter centered at $\lambda 4649$ which had a transmission width (FWHM) of $\sim 30 \AA$ A. The photons detected were synchronously co-added at the $\mathrm{X}$-ray pulsar rate in a multichannel scaler with channels $40 \mathrm{~ms}$ wide. No significant pulsations were observed to a level of 2 percent $(3 \sigma)$. This observation provided an easy check on the suggestion (Rees and Angel 1973) that the optical pulsing might be confined to the $\mathrm{C}$ III-N III emission-line complex at $\lambda 4650$.

TABLE 1

\begin{tabular}{|c|c|c|c|c|}
\hline $\begin{array}{c}\text { Beginning of } \\
\text { Light Curve } \\
\left(\mathrm{JD}_{\odot}+2,441,900\right)\end{array}$ & $\begin{array}{l}\text { Inte- } \\
\text { gra- } \\
\text { tion } \\
\text { Time } \\
\text { (s) }\end{array}$ & $\begin{array}{l}\text { Length } \\
\text { (s) }\end{array}$ & $\begin{array}{l}\text { Orbital } \\
\text { Phase } \\
\text { (midpoint) }\end{array}$ & $\underset{(\%)}{F}$ \\
\hline $\begin{array}{l}26.708635 \ldots \ldots \ldots \\
26.723289 \ldots \ldots \ldots \\
27.741612 \ldots \ldots \ldots \\
28.633683 \ldots \ldots \\
19.68663 \ldots \ldots \\
20.735656 \ldots \ldots \\
21.655464 \ldots \ldots \\
28.73853 \ldots \ldots\end{array}$ & $\begin{array}{l}4 \\
4 \\
4 \\
4 \\
\frac{1}{4} \\
\frac{1}{4} \\
\frac{1}{4} \\
\frac{1}{4}\end{array}$ & $\begin{array}{l}1252 \\
2276 \\
1876 \\
5404 \\
2287 \\
1247 \\
3027 \\
2120\end{array}$ & $\begin{array}{l}0.225 \\
0.229 \\
0.835 \\
0.372 \\
0.098 \\
0.712 \\
0.259 \\
0.422\end{array}$ & $\begin{array}{l}1.12 \\
1.09 \\
0.81 \\
1.59 \\
\ldots \\
\ldots \\
\ldots \\
\ldots\end{array}$ \\
\hline
\end{tabular}

\section{DISCUSSION}

These observations reveal flickering in the $\mathrm{HZ} \mathrm{Her}$ system, following the 1973 August $24 \mathrm{X}$-ray turn-on, which is present from orbital phase $0.2 \leq \Phi \leq 0.7$ with an apparent maximum in amplitude near $\Phi=0.5$. This correlation with phase rules out a hot spot in a disk of material surrounding the $\mathrm{X}$-ray source as the origin of the flickering. Warner and Peters (1972) have calculated the size and location of such a hot spot for systems of various mass ratios. Their results predict maximum flickering in HZ Her orbital phases $0.75 \leq \Phi \leq 0.00$, where the range is virtually independent of mass ratios $\left(0.01 \leq M_{\mathrm{X}} / M_{\mathrm{HZ}} \leq 10.0\right)$ but allows for increased thermal escape velocities from the inner Lagrangian point due to X-ray heating, and little or no flickering at phases $0.25 \leq \Phi \leq 0.50$. We emphasize that these observations do not rule out models involving substantial optical emission from an entire disk of material around the X-ray source. Indeed, such systems would be expected to show maximum flickering at $\Phi=0.5$.

The apparent maximum in the amplitude of the flickering at $\Phi=0.5$, together with the observations of Boynton et al. (1973), suggest that the "third light" is associated with the X-ray heating of material close to or actually in the upper atmosphere of the hot side of HZ Her. We note that the time scales of $15-300 \mathrm{~s}$ are consistent with thermal cooling times of 3-30 s obtained by Alme and Wilson (1973) for photospheric material heated by X-rays.

Tananbaum et al. (1973) report that the turn-on time for the high state of the 35-day cycle on Her X-1 occurred UT 1973 August $24.7 \pm 1.0$; therefore, all of our observations were obtained during the X-ray on cycle.

Observations during the ofF part of the $35^{\mathrm{d}} \mathrm{X}$-ray cycle would be useful. The absence of flickering during X-ray ofF would imply that the hard $(2-20 \mathrm{keV})$ pulsed X-rays are responsible for the heating associated with the flickering, although not for the bulk heating which requires a steady energy source (Avni et al. 1973). Presence of flickering during X-ray ofF would support those models using precession of the neutron-star spin axis to account for this behavior, or else some other flickering mechanism would have to be invoked.

We plan to continue our investigations of the flickering behavior in $\mathrm{HZ} \mathrm{Her} \mathrm{in} \mathrm{order} \mathrm{to} \mathrm{obtain} \mathrm{more} \mathrm{com-}$ plete phase coverage, and we urge other observers capable of simultaneous dual-channel observations to monitor this system also.

This work was supported in part by NASA grant NGR 44-012-209 and grants GP-25901 and GP-41796 from the National Science Foundation. The authors would like to thank Mr. Alan Kiplinger for his generous assistance at the telescope and his advice concerning the data reduction. The clarifying suggestions of the referee are very much appreciated. 


\section{REFERENCES}

Alme, M. L., and Wilson, J. R. 1973, preprint.

I Avni, Y., Bahcall, J. N., Joss, P. C., Bahcall, N. A., Lamb, F. K., Pethick, C. J., and Pines, D. 1973, Nature Phys. Sci., 246, 36.

Bopp, B. W., Grupsmith, G., McMillan, R. S., Vanden Bout, P., and Wootten, H. A. 1973, A p. J. (Letters), 186, L123.

Bopp, B. W., Grupsmith, G., and Vanden Bout, P. 1972, Ap.J. (Letters), 178, L5.

Boynton, P. E., Cantera, R., Crosa, L., Deeter, J., and Gerend, D. 1973, Ap.J., 186, 617 .

Davidsen, A., Henry, J. P., Middleditch, J., and Smith, H. E. 1972, A p.J. (Letters), 177, L97.

Groth, E. J., and Nelson, M. R. 1972, Ap.J. (Letters), 178, L111.
Joss, P. C., Avni, Y., and Bahcall, J. N. 1973, Ap. J.. 186, 767.

Nather, R. E. 1972, thesis, University of Cape Town.

Pringle, O. O. 1973, Nature Phys. Sci., 243, 90.

Rees, M., and Angel, J. R. P. 1973, private communication.

Robinson, E. L., and Warner, B. 1972, M.N.R.A.S., 157, 85.

Smak, J. I. 1971, Acta Astr., 21, 15.

Strittmatter, P. A., Scott, J., Whelan, J., Wickramasinghe, D. T., and Woolf, N. J. 1973, A str. and A p., 25, 275.

Tananbaum, H., Schreier, E., Levinson, R., Giacconi, R., Gursky, H., Kellogg, E., and Matilsky, T. 1973, IAU Circ., No. 2578.

Warner, B., and Nather, R. E. 1971, M.N.R.A.S., 152, 219.

Warner, B., and Peters, W. L. 1972, M.N.R.A.S., 160, 15. 